\title{
CONSTRUCTIONS OF TRACE ZERO SYMMETRIC STOCHASTIC MATRICES FOR THE INVERSE EIGENVALUE PROBLEM*
}

\author{
ROBERT REAMS
}

\begin{abstract}
In the special case of where the spectrum $\sigma=\left\{\lambda_{1}, \lambda_{2}, \lambda_{3}, 0,0, \ldots, 0\right\}$ has at most three nonzero eigenvalues $\lambda_{1}, \lambda_{2}, \lambda_{3}$ with $\lambda_{1} \geq 0 \geq \lambda_{2}>\lambda_{3}$, and $\lambda_{1}+\lambda_{2}+\lambda_{3}=0$, the inverse eigenvalue problem for symmetric stochastic $n \times n$ matrices is solved. Constructions are provided for the appropriate matrices where they are readily available. It is shown that when $n$ is odd it is not possible to realize the spectrum $\sigma$ with an $n \times n$ symmetric stochastic matrix when $\lambda_{3} \neq 0$ and $\frac{3}{2 n-3}>\frac{\lambda_{2}}{\lambda_{3}} \geq 0$, and it is shown that this bound is best possible.
\end{abstract}

Key words. Inverse eigenvalue problem, Symmetric stochastic matrix, Symmetric nonnegative matrix, Distance matrix.

AMS subject classifications. 15A18, 15A48, 15A51, 15A 57

1. Introduction. Let $e_{1}, \ldots, e_{n}$ denote the standard basis in $\mathbb{R}^{n}$, so $e_{i}$ denotes the vector with a 1 in the $i$ th position and zeroes elsewhere. We will denote by $e$ the vector of all ones, i.e. $e=[1,1, \ldots, 1]^{T} \in \mathbb{R}^{n}$. A matrix $A \in \mathbb{R}^{n \times n}$ is said to be stochastic when all of its entries are nonnegative and all its row sums are equal to 1 , i.e. $A$ has right eigenvector $e$ corresponding to the eigenvalue 1 . We will be concerned with symmetric stochastic matrices, so that these matrices are in fact doubly stochastic. Also, the eigenvalues will necessarily be real. If $A \in \mathbb{R}^{n \times n}$ is nonnegative, has eigenvalue $\lambda_{1}>0$ corresponding to the right eigenvector $e$ then $\frac{1}{\lambda_{1}} A$ is stochastic, and for convenience we will state our results in the form, for example, of a matrix $A$ having eigenvector $e$ corresponding to $1+\epsilon$, where the spectrum $\sigma=$ $\{1+\epsilon,-1,-\epsilon, 0,0, \ldots, 0\}$, with $0 \leq \epsilon \leq 1$. We will say that $\sigma=\left\{\lambda_{1}, \ldots, \lambda_{n}\right\} \subset \mathbb{R}$ is realized as the spectrum of a matrix $A$ in the event that the $n \times n$ matrix $A$ has eigenvalues $\lambda_{1}, \ldots, \lambda_{n}$.

The nonnegative inverse eigenvalue problem is to find necessary and sufficient conditions that the elements of the set $\sigma=\left\{\lambda_{1}, \ldots, \lambda_{n}\right\} \subset \mathbb{C}$ are the eigenvalues of a matrix with nonnegative entries. This problem is currently unsolved except in special cases [1], [7], [8], [9], [10]. The restriction of this problem to symmetric nonnegative matrices for which the eigenvalues $\lambda_{1}, \ldots, \lambda_{n}$ satisfy $\lambda_{1} \geq 0 \geq \lambda_{2} \geq \cdots \geq \lambda_{n}$ is solved in [3], where it is shown that the only necessary and sufficient condition is that $\sum_{i=1}^{n} \lambda_{i} \geq 0$. Distance matrices are necessarily symmetric, nonnegative, have trace zero, and must have $\lambda_{1} \geq 0 \geq \lambda_{2} \geq \cdots \geq \lambda_{n}$, although these are not sufficient conditions to be a distance matrix. We conjectured in [6], after solving numerous special cases including $n=2,3,4,5,6$, that the only necessary and sufficient conditions for the existence of a distance matrix with a given $\sigma$ is that $\lambda_{1} \geq 0 \geq \lambda_{2} \geq \cdots \geq \lambda_{n}$ and $\sum_{i=1}^{n} \lambda_{i}=0$. Distance matrices with eigenvector e were previously studied in [5],

* Received by the editors on 13 October 2001. Final manuscript accepted for publication on 4 September 2002. Handling Editor: Tom Laffey.

$\dagger$ Department of Mathematics, National University of Ireland Galway, Galway, Ireland (robert.reams@nuigalway.ie). 
although not in the context of these matrices eigenvalues. Bounds on the eigenvalues of symmetric stochastic matrices are given in [2] and [4], although they do not provide a restriction on the eigenvalues for the matrices under consideration here.

In Section 2 we provide an explicit construction of an $n \times n$ symmetric stochastic matrix which realizes the spectrum $\{2,-1,-1,0,0, \ldots, 0\}$, followed by showing that it is not possible to realize the spectrum $\{1,-1,0,0, \ldots, 0\}$ with a symmetric stochastic matrix when $n$ is odd, although it is possible to realize this spectrum when $n$ is even. In Section 3 we provide explicit constructions of symmetric stochastic matrices to realize $\{1+\epsilon,-1,-\epsilon, 0,0, \ldots, 0\}$, for $1 \geq \epsilon \geq 0$, when $n$ is even. We then show that it is not possible to realize this spectrum with a symmetric stochastic matrix when $\frac{3}{2 n-3}>\epsilon \geq 0$, and $n$ is odd. Although we can realize this spectrum with a symmetric stochastic matrix when $1 \geq \epsilon \geq \frac{3}{2 n-3}$, and $n$ is odd. In the latter case we do not provide an explicit construction, instead making use of the Intermediate Value Theorem in several variables.

2. Freedom and restrictions on spectra. Lemma 2.1 will be used to establish that the matrix under consideration is nonnegative.

Lemma 2.1. Let $A=\left(a_{i j}\right) \in \mathbb{R}^{n \times n}$ be a symmetric matrix with eigenvalues $\lambda_{1}, \ldots, \lambda_{n}$ which satisfy $\lambda_{1} \geq 0 \geq \lambda_{2} \geq \cdots \geq \lambda_{n}$. Suppose that $A$ has eigenvector $e$ corresponding to $\lambda_{1}$, and that $A$ has all diagonal entries equal to zero. Then $A$ is a nonnegative matrix.

Proof. Write $A=\lambda_{1} \frac{e e^{T}}{n}+\lambda_{2} u_{2} u_{2}^{T}+\cdots+\lambda_{n} u_{n} u_{n}^{T}$, where $u_{2}, \ldots, u_{n}$ are unit eigenvectors corresponding to $\lambda_{2}, \ldots, \lambda_{n}$, respectively. Then $-2 a_{i j}=\left(e_{i}-e_{j}\right)^{T} A\left(e_{i}-\right.$ $\left.e_{j}\right)=\lambda_{2}\left(\left(e_{i}-e_{j}\right)^{T} u_{2}\right)^{2}+\cdots+\lambda_{n}\left(\left(e_{i}-e_{j}\right)^{T} u_{n}\right)^{2} \leq 0$, for all $i, j, 1 \leq i, j \leq n$.

Our next two theorems give some foretaste of what is and isn't possible with the inverse eigenvalue problem for symmetric nonnegative matrices. Note first that a $3 \times 3$ symmetric nonnegative matrix with eigenvector $e$ and of trace zero must be a nonnegative scalar multiple of $A=e e^{T}-I$, which has spectrum $\sigma=\{2,-1,-1\}$.

TheOREm 2.2. Let $\sigma=\{2,-1,-1,0,0, \ldots, 0\}$. Then $\sigma$ can be realized as the spectrum of a symmetric nonnegative matrix $A \in \mathbb{R}^{n \times n}$ with eigenvector e corresponding to 2 , for $n \geq 3$.

Proof. Let $u=\left[u_{1}, \ldots, u_{n}\right]^{T}, v=\left[v_{1}, \ldots, v_{n}\right]^{T} \in \mathbb{R}^{n}$ be given by $u_{j}=\sqrt{\frac{2}{n}} \cos \theta_{j}$, $v_{j}=\sqrt{\frac{2}{n}} \sin \theta_{j}$, where $\theta_{j}=\frac{2 \pi}{n} j$, for $1 \leq j \leq n$. Then $A=2 \frac{e e T}{n}-u u^{T}-v v^{T}$ is symmetric and has zero diagonal entries by construction. Since the roots of $x^{n}-1$ are $e^{\frac{2 \pi i}{n} j}, 1 \leq j \leq n$, then (coefficient of $x^{n-1}$ ) $=0=\sum_{j=1}^{n}-e^{\frac{2 \pi i}{n} j}$ and (coefficient of $\left.x^{n-2}\right)=0=\sum_{j, k=1, j \neq k}^{n} e^{\frac{2 \pi i}{n} j} e^{\frac{2 \pi i}{n} k}=\left(\sum_{j=1}^{n} e^{\frac{2 \pi i}{n} j}\right)^{2}-\sum_{j=1}^{n} e^{\frac{2 \pi i}{n} 2 j}$. It follows that $\sum_{j=1}^{n} \cos \theta_{j}=\sum_{j=1}^{n} \sin \theta_{j}=\sum_{j=1}^{n} \sin 2 \theta_{j}=0$, which tells us that $u^{T} e=v^{T} e=$ $u^{T} v=0$, respectively. It also follows that $\sum_{j=1}^{n} \cos 2 \theta_{j}=0$, so that $\sum_{j=1}^{n} \cos ^{2} \theta_{j}-$ $\sum_{j=1}^{n} \sin ^{2} \theta_{j}=0$. We also know that $\sum_{j=1}^{n} \cos ^{2} \theta_{j}+\sum_{j=1}^{n} \sin ^{2} \theta_{j}=n$, so we can conclude that $\sum_{j=1}^{n} \cos ^{2} \theta_{j}=\sum_{j=1}^{n} \sin ^{2} \theta_{j}=\frac{n}{2}$. This tells us that $u$ and $v$ are unit vectors. Thus $A$ has spectrum $\sigma$ and is nonnegative from Lemma 2.1. $\square$

TheOREM 2.3. Let $\lambda_{1}>0$ and $\sigma=\left\{\lambda_{1},-\lambda_{1}, 0, \ldots, 0\right\}$. Then $\sigma$ cannot be realized as the spectrum of an $n \times n$ symmetric nonnegative matrix with eigenvector 
e corresponding to $\lambda_{1}$, when $n$ is odd.

Proof. Suppose $A$ is a matrix of the given form that realizes $\sigma$. Write $A=$ $\lambda_{1} \frac{e e^{T}}{n}-\lambda_{1} u u^{T}$, where $u=\left[u_{1}, \ldots, u_{n}\right]^{T} \in \mathbb{R}^{n},\|u\|=1$ and $u^{T} e=0 . \quad A$ has trace zero and is nonnegative by hypothesis so all diagonal entries are zero, and thus $0=\frac{\lambda_{1}}{n}-\lambda_{1} u_{i}^{2}$, for $1 \leq i \leq n$. But then $u_{i}=\frac{ \pm 1}{\sqrt{n}}$ and $0=\sum_{i=1}^{n} u_{i}=\sum_{i=1}^{n} \frac{ \pm 1}{\sqrt{n}}$, which is not possible when $n$ is odd.

REMARK 2.4. The same $\sigma$ of Theorem 2.3 can be realized by an $n \times n$ symmetric nonnegative matrix with eigenvector $e$ corresponding to $\lambda_{1}$ when $n$ is even, by taking in the proof the unit vector $u=\frac{1}{\sqrt{n}}[1,1, \ldots, 1,-1,-1, \ldots,-1]^{T} \in \mathbb{R}^{n}$.

3. At most three nonzero eigenvalues. The idea in Remark 2.4 can be extended to the case where $n$ is a multiple of 4 for the case of at most three nonzero eigenvalues.

Theorem 3.1. Let $\sigma=\{1+\epsilon,-1,-\epsilon, 0,0, \ldots, 0\}$, where $1 \geq \epsilon \geq 0$. Then $\sigma$ can be realized by a symmetric nonnegative matrix $A \in \mathbb{R}^{n \times n}$ with eigenvector $e$ corresponding to $1+\epsilon$, when $n=4 m$ for some $m$.

Proof. Let $u, v \in \mathbb{R}^{n}$ be given by

$$
\begin{aligned}
& u=\frac{1}{\sqrt{n}}[1,1, \ldots, 1,1,1, \ldots, 1,-1,-1, \ldots,-1,-1,-1, \ldots,-1]^{T}, \\
& v=\frac{1}{\sqrt{n}}[1,1, \ldots, 1,-1,-1, \ldots,-1, \quad 1, \quad 1, \ldots, 1,-1,-1, \ldots,-1]^{T} .
\end{aligned}
$$

Then $\frac{e}{\sqrt{n}}, u$ and $v$ are orthonormal. Let $A=\frac{(1+\epsilon)}{n} e e^{T}-u u^{T}-\epsilon v v^{T}$, and note that all the diagonal entries of $A$ are zero. $\square$

However, the way we deal with the remaining cases for $n$ even is somewhat more complicated.

TheOREM 3.2. Let $\sigma=\{1+\epsilon,-1,-\epsilon, 0,0, \ldots, 0\}$, where $1 \geq \epsilon \geq 0$. Then $\sigma$ can be realized by a symmetric nonnegative matrix $A \in \mathbb{R}^{n \times n}$ with eigenvector $e$ corresponding to $1+\epsilon$, when $n=4 m+2$ for some $m$.

Proof. Let $u=\left[u_{1}, \ldots, u_{n}\right]^{T}, v=\left[v_{1}, \ldots, v_{n}\right]^{T} \in \mathbb{R}^{n}$, and $A=(1+\epsilon) \frac{e e^{T}}{n}-$ $u u^{T}-\epsilon v v^{T}$. We will require that $0=\frac{1+\epsilon}{n}-u_{i}^{2}-\epsilon v_{i}^{2}$, for $1 \leq i \leq n$, so that $A$ has all zero diagonal entries. Let $u_{i}=\sqrt{\frac{1+\epsilon}{n}} \cos \theta_{i}$ and $v_{i}=\sqrt{\frac{1+\epsilon}{n \epsilon}} \sin \theta_{i}$, where the $\theta_{i}$ 's are chosen below. Our $\theta_{i}$ 's must satisfy $\sum_{i=1}^{n} \cos \theta_{i}=\sum_{i=1}^{n} \sin \theta_{i}=$ $\sum_{i=1}^{n} \cos \theta_{i} \sin \theta_{i}=0$. So that $u$ and $v$ are unit vectors we must have $\sum_{i=1}^{n} \cos ^{2} \theta_{i}=$ $\frac{n}{1+\epsilon}$ and $\sum_{i=1}^{n} \sin ^{2} \theta_{i}=\frac{n \epsilon}{1+\epsilon}$, which will be achieved if $\sum_{i=1}^{n} \cos ^{2} \theta_{i}-\sum_{i=1}^{n} \sin ^{2} \theta_{i}=$ $\frac{n(1-\epsilon)}{1+\epsilon}$, and $\sum_{i=1}^{n} \cos ^{2} \theta_{i}+\sum_{i=1}^{n} \sin ^{2} \theta_{i}=n$. So for any given $\epsilon \in[0,1]$ we also require for our choice of $\theta_{i}$ 's that $\sum_{i=1}^{n} \cos 2 \theta_{i}=\frac{n(1-\epsilon)}{1+\epsilon}$. plane.

Let $0 \leq \delta<\frac{2 \pi}{n}$, and choose the angles $\theta_{i}, 1 \leq i \leq n$, around the origin in the

Let $\theta_{i}$ for $1 \leq i \leq m$ be, respectively, the 1st quadrant angles

$$
\frac{2 \pi}{n}-\delta, 2\left(\frac{2 \pi}{n}-\delta\right), \ldots,(m-1)\left(\frac{2 \pi}{n}-\delta\right), m\left(\frac{2 \pi}{n}-\delta\right) \text {. }
$$

Let $\theta_{i}$ for $m+1 \leq i \leq 2 m$ be, respectively, the 2 nd quadrant angles

$$
(m+1) \frac{2 \pi}{n}+m \bar{\delta},(\bar{m}+2) \frac{2 \pi}{n}+(m-1) \delta, \ldots,(2 m-1) \frac{2 \pi}{n}+2 \delta, 2 m \frac{2 \pi}{n}+\delta .
$$

Let $\theta_{2 m+1}=\pi$.

Let $\theta_{i}$ for $2 m+2 \leq i \leq 3 m+1$ be, respectively, the 3rd quadrant angles

$-2 m \frac{2 \pi}{n}-\delta,-(2 m-1) \frac{2 \pi}{n}-2 \delta, \ldots,-(m+2) \frac{2 \pi}{n}-(m-1) \delta,-(m+1) \frac{2 \pi}{n}-m \delta$. 
Let $\theta_{i}$ for $3 m+2 \leq i \leq 4 m+1$ be, respectively, the 4th quadrant angles

Let $\theta_{4 m+2}=0$.

$$
-m\left(\frac{2 \pi}{n}-\delta\right),-(m-1)\left(\frac{2 \pi}{n}-\delta\right), \ldots,-2\left(\frac{2 \pi}{n}-\delta\right),-\left(\frac{2 \pi}{n}-\delta\right) \text {. }
$$

For each $\theta_{i}$ there is a $\theta_{i}+\pi$ in the above list, by pairing off 1 st quadrant angles with appropriate 3rd quadrant angles, and pairing 2nd quadrant angles with appropriate 4 th quadrant angles. Therefore since $\cos \left(\theta_{i}+\pi\right)=-\cos \theta_{i}$ we conclude that $\sum_{i=1}^{n} \cos \theta_{i}=0$.

For each $\theta_{i} \neq 0$ and $\theta_{i} \neq \pi$ in the above list we can pair off any $\theta_{i}$ with a corresponding $-\theta_{i}$ and conclude that $\sum_{i=1}^{n} \sin \theta_{i}=0$.

Similarly, $\sum_{i=1}^{n} \sin 2 \theta_{i}=0$.

Pairing off each $\theta_{i}$ with a $\theta_{i}+\pi$ in the same way as before, and since $\cos 2 \theta_{i}=$ $\cos \left(2 \theta_{i}+2 \pi\right)$, we must have

$$
\sum_{i=1}^{n} \cos 2 \theta_{i}=2+\sum_{1 \mathrm{st}, 2 \mathrm{nd}, 3 \mathrm{rd}, 4 \text { th quadrant }} \cos 2 \theta_{i}=2+2 \sum_{1 \mathrm{st}, 4 \text { th quadrant }} \cos 2 \theta_{i} .
$$

Because we can pair off each $\theta_{i}$ in the 1 st quadrant with each $-\theta_{i}$ in the 4 th quadrant $\sum_{i=1}^{n} \cos 2 \theta_{i}=2+4 \sum_{1 \text { st quadrant }} \cos 2 \theta_{i}$. Next using the trigonometric formula

$$
\sum_{j=0}^{n} \cos j \alpha=\frac{\sin \left(\frac{n+1}{2} \alpha\right) \cos \left(\frac{n}{2} \alpha\right)}{\sin \frac{\alpha}{2}}
$$

(found in [11], for example), where $\alpha=2\left(\frac{2 \pi}{n}-\delta\right)$ and some simplification we obtain that $\sum_{i=1}^{n} \cos 2 \theta_{i}=2 \frac{\sin ((2 m+1) \delta)}{\sin \left(\frac{\pi}{2 m+1}-\delta\right)}$. Let $f(\delta)=2 \frac{\sin ((2 m+1) \delta)}{\sin \left(\frac{\pi}{2 m+1}-\delta\right)}$, then $f(0)=0$ and $\lim _{\delta \rightarrow \frac{2 \pi}{n}} f(\delta)=n$, and notice that $f$ is continuous on the interval $\left[0, \frac{2 \pi}{n}\right)$. Also, since $0 \leq \epsilon \leq 1$ we have that $0 \leq \frac{n(1-\epsilon)}{1+\epsilon} \leq n$, but then by the Intermediate Value Theorem for each $\epsilon \in(0,1]$ there is a $\delta \in\left[0, \frac{2 \pi}{n}\right)$ such that $f(\delta)=\frac{n(1-\epsilon)}{1+\epsilon}$. The case $\epsilon=0$ and $\delta=\frac{2 \pi}{n}$ is covered by the remark after Theorem 2.3. $\square$

In order to deal with the case where $n$ is odd we will improve on Theorem 2.3.

Theorem 3.3. Let $\sigma=\{1+\epsilon,-1,-\epsilon, 0,0, \ldots, 0\}$, where $\frac{3}{2 n-3}>\epsilon \geq 0$, and $n \geq 3$. Then $\sigma$ cannot be realized by a symmetric nonnegative matrix $A \in \mathbb{R}^{n \times n}$ with eigenvector e corresponding to $1+\epsilon$, when $n=2 m+1$ for some $m$.

Proof. $\sigma$ is realizable when $\epsilon=1$ from Theorem 2.2. We wish to determine the minimum value of $\epsilon$ for which it is possible to construct a matrix of the desired form. Using the same notation as in the proof of Theorem 3.2 we wish to determine the minimum value of $\epsilon$ as a function of $\theta_{1}, \ldots, \theta_{n}$ subject to the three constraints $\sum_{i=1}^{n} \cos \theta_{i}=\sum_{i=1}^{n} \sin \theta_{i}=0$ and $\sum_{i=1}^{n} \cos \theta_{i} \sin \theta_{i}=0$ (we know from Theorem 2.3 that $\epsilon>0)$. Observe that finding the minimum $\epsilon$ is equivalent to finding the maximum value of the function $\frac{n}{1+\epsilon}=F\left(\theta_{1}, \ldots, \theta_{n}\right)=\sum_{i=1}^{n} \cos ^{2} \theta_{i}$ subject to the three constraints. For the moment let us determine the maximum value of $F$ subject to the one constraint $\sum_{i=1}^{n} \cos \theta_{i}=0$. Let $\lambda$ denote the Lagrange multiplier so that $-2 \cos \theta_{i} \sin \theta_{i}-\lambda \sin \theta_{i}=0$, i.e. $\sin \theta_{i}\left(2 \cos \theta_{i}+\lambda\right)=0$, for each $i, 1 \leq i \leq n$. Then 
for each $i$ we have $\sin \theta_{i}=0$ or else $\cos \theta_{i}=-\lambda / 2$. We cannot have all $\theta_{i}$ 's equal to 0 or $\pi$, since then we would not have $\sum_{i=1}^{n} \cos \theta_{i}=0$, because $n$ is odd. Suppose now that $k$ of the $\cos \theta_{i}$ 's are equal, but not equal to \pm 1 , then $k \cos \theta_{i}= \pm 1 \pm 1 \cdots$. Then in order to maximize $F$ we must have as many \pm 1 's as possible, thus we must have $k=2$ with $\cos \theta_{p}=\cos \theta_{q}$ (say) and the remaining $\theta_{i}$ 's all either 0 or $\pi$. Then $2 \cos \theta_{i}=1$ or -1 , and there are two possibilities:

Case 1: $\cos \theta_{p}=\cos \theta_{q}=\frac{1}{2}$ with $m-1$ of the remaining $\cos \theta_{i}$ 's equal to 1 and the other $m$ of the $\cos \theta_{i}$ 's equal to -1 .

Case 2: $\cos \theta_{p}=\cos \theta_{q}=-\frac{1}{2}$ with $m-1$ of the remaining $\cos \theta_{i}$ 's equal to -1 and the other $m$ of the $\cos \theta_{i}$ 's equal to 1 .

In either case the maximum value of $F$ is $\frac{1}{2}+2 m-1=n-\frac{3}{2}$, in which case $\epsilon=\frac{3}{2 n-3}$.

Notice now that this maximum value for $F$ can be just as easily achieved when $\theta_{p}=-\theta_{q}$ and that then $\sum_{i=1}^{n} \sin \theta_{i}=0$ and $\sum_{i=1}^{n} \cos \theta_{i} \sin \theta_{i}=0$. So $\epsilon$ has in effect been minimized subject to all three constraints. $\square$

Corollary 3.4. Let $\sigma=\{1+\epsilon,-1,-\epsilon, 0, \ldots, 0\}$, where $1 \geq \epsilon \geq \frac{3}{2 n-3}$, and $n \geq 3$. Then $\sigma$ can realized as the spectrum of a symmetric nonnegative matrix $A \in \mathbb{R}^{n \times n}$ with eigenvector e corresponding to $1+\epsilon$, when $n$ is odd.

Proof. Let $F\left(\theta_{1}, \ldots, \theta_{n}\right)=\sum_{j=1}^{n} \cos ^{2} \theta_{j}$. Then

$$
F\left(\frac{2 \pi}{n} 1, \frac{2 \pi}{n} 2, \ldots, \frac{2 \pi}{n}(n-1), \frac{2 \pi}{n} n\right)=\frac{n}{2},
$$

and

$$
F\left(\frac{2 \pi}{3},-\frac{2 \pi}{3}, 0,0, \ldots, 0, \pi, \pi, \ldots, \pi\right)=n-\frac{3}{2}
$$

Moreover, $F$ is continuous as a function of $\cos \theta_{1}, \ldots, \cos \theta_{n}$, particularly on the following intervals for the $\cos \theta_{i}$ 's:

For $n=4 k+3$ let the $\cos \theta_{i}$ 's satisfy $\cos \frac{2 \pi(k+1)}{n} \leq \cos \theta_{k+1} \leq \cos \frac{2 \pi}{3}$ and $\cos \frac{2 \pi(3 k+3)}{n} \leq \cos \theta_{3 k+3} \leq \cos \frac{2 \pi}{3}$. Also, let $\cos \frac{2 \pi}{n} j \leq \cos \theta_{j} \leq \cos 0$ for $1 \leq j \leq k$ and $3 k+4 \leq j \leq 4 k+3$, and let $\cos \pi \leq \cos \theta_{j} \leq \cos \frac{2 \pi}{n} j$ for $k+2 \leq j \leq 3 k+2$ except that $\cos \theta_{2 k+2}=\cos \pi$.

For $n=4 k+1$ let the $\cos \theta_{i}$ 's satisfy $\cos \frac{2 \pi(k+1)}{n} \leq \cos \theta_{k+1} \leq \cos \frac{2 \pi}{3}$ and $\cos \frac{2 \pi(3 k+2)}{n} \leq \cos \theta_{3 k+2} \leq \cos \frac{2 \pi}{3}$. Also, let $\cos \frac{2 \pi}{n} j \leq \cos \theta_{j} \leq \cos 0$ for $1 \leq j \leq k$ and $3 k+3 \leq j \leq 4 k+1$, and let $\cos \pi \leq \cos \theta_{j} \leq \cos \frac{2 \pi}{n} j$ for $k+2 \leq j \leq 3 k+1$ except that $\cos \theta_{1}=\cos 0$.

For each $\epsilon$ such that $1 \geq \epsilon \geq \frac{3}{2 n-3}$ we have $\frac{n}{2} \leq \frac{n}{1+\epsilon} \leq n-\frac{3}{2}$, then from the Intermediate Value Theorem for real valued functions of several variables it follows that for each $\epsilon \in\left[\frac{3}{2 n-3}, 1\right]$ there is a $\left(\theta_{1}, \ldots, \theta_{n}\right)$ such that $F\left(\theta_{1}, \ldots, \theta_{n}\right)=\frac{n}{1+\epsilon}$.

The author does not see a natural extension of the above methods to deal with the case of at most four nonzero eigenvalues.

Acknowledgement. I am grateful to Ron Smith and Charles R. Johnson for some interesting conversations. 


\section{REFERENCES}

[1] M. Boyle and D. Handelman. The spectra of nonnegative matrices via symbolic dynamics. Annals of Mathematics, 133:249-316, 1991.

[2] M. Fiedler. Bounds for eigenvalues of doubly stochastic matrices. Linear Algebra and Its Applications, 5:299-310, 1972.

[3] M. Fiedler. Eigenvalues of nonnegative symmetric matrices. Linear Algebra and Its Applications, 9:119-142, 1974.

[4] M. Fiedler. Additive compound matrices and an inequality for eigenvalues of symmetric stochastic matrices. Czechoslovak Mathematical Journal, 24(99):392-402, 1974.

[5] T.L. Hayden and P. Tarazaga. Distance matrices and regular figures. Linear Algebra and its Applications, 195:9-16, 1993.

[6] T.L. Hayden, R. Reams, and J. Wells. Methods for constructing distance matrices and the inverse eigenvalue problem. Linear Algebra and Its Applications, 295:97-112, 1999.

[7] T.J. Laffey and E. Meehan. A characterization of trace zero nonnegative $5 \times 5$ matrices. Linear Algebra and Its Applications, 302/303:295-302, 1999.

[8] R. Loewy and D. London. A note on an inverse problem for nonnegative matrices. Linear and Multilinear Algebra, 6:83-90, 1978.

[9] H. Minc. Nonnegative Matrices. John Wiley and Sons, New York, 1988.

[10] R. Reams. An inequality for nonnegative matrices and the inverse eigenvalue problem. Linear and Multilinear Algebra, 41:367-375, 1996.

[11] D.O. Shklarsky, N.N. Chentzov, and I.M. Yaglom. The USSR Olympiad Problem Book. Dover Publications, Inc., New York, 1993. 